\title{
Error Estimators for Proper Generalized Decomposition (PGD) in Time Dependent Electromagnetic Field Problems
}

\author{
F. Müller ${ }^{1 *}$, T. Henneron ${ }^{2}$, S. Clénet ${ }^{3}$ and K. Hameyer ${ }^{1}$ \\ ${ }^{1}$ Institute of Electrical Machines (IEM), RWTH Aachen University, Aachen, Germany \\ ${ }^{2}$ L2EP, Université de Lille, Lille, France \\ ${ }^{3}$ L2EP, Ecole Nationale Supérieure d'Arts et Métiers, Lille, France \\ Corresponding author: F. Müller (email: fabian.mueller@iem.rwth-aachen.de
}

\begin{abstract}
Due to fine discretization in space and time, the simulation of transient electromagnetic phenomena results in a large system of equations. To cope with this computational effort, model order reduction techniques can be employed. To assess the accuracy of the solution of the reduced model, an error estimation is crucial. A commonly used approach consists of the evaluation of the deviation between the reduced and the full model. This yields a loss of the a-priori property of the Proper Generalized Decomposition. To overcome this problem two a-priori criteria are presented in this paper.
\end{abstract}

Index Terms-Error criteria, finite element method (FEM), model order reduction (MOR), proper generalized decomposition (PGD).

\section{INTRODUCTION}

$\mathbf{L}$ ARGE scale finite element models arise from e.g. time dependent electromagnetic field problems, due to the skin depths of the eddy currents. On the one hand, to properly model eddy currents, the conducting regions have to be accurately discretized in space. On the other hand the time interval has to be sampled accurately to consider all transient effects. The resulting computational effort of these transient simulations can be reduced by model order reduction (MOR). The reduction techniques can be distinguished in two classes, namely a-posteriori and a-priori methods. One well known aposteriori method is the Proper Orthogonalized Decomposition (POD), which is based on collecting snapshots of the reference system to calculate a reduced representation. A-priori methods such as the Proper Generalized Decomposition (PGD) method construct a reduced order model without any previously obtained solutions [1]. The PGD has been applied to different problems in mechanics [1]-[4] and electromagnetics [5]-[10], [12], [13] and represents a desirable strategy to solve engineering problems. While different error criteria for a-posteriori methods have been formulated [6], a reasonable criterium for a-priori methods has not been stated yet. To maintain the a-priori property of the PGD, an a-priori error criterium is presented in the following.

\section{Magnetoquasistatic Problem}

To solve the magnetoquasistatic field problem, the Finite Element Method (FEM) with the magnetic vector potential $\boldsymbol{A}$ is employed (1). The problem consists of a domain with unary boundary conditions and a conducting subdomain which allows eddy currents.

$$
\nabla \times \nu(\nabla \times \boldsymbol{A}(t))+\frac{\sigma \partial \boldsymbol{A}(t)}{\partial t}=\boldsymbol{J}(t)
$$

\section{Proper Generalized Decomposition}

\section{A. Basic approach}

The basic principle of the PGD is to decompose the solution of a linear partial differential equation (PDE) into a sum of $m$ tensor products (2) [1], [2], [9].

$$
\boldsymbol{A}(\mathrm{x}, \mathrm{t}) \approx \sum_{\mathrm{i}=1}^{\mathrm{m}} \boldsymbol{R}_{\mathrm{i}}(x) S_{\mathrm{i}}(t)
$$

$\boldsymbol{R}(x)$ is the space related part of the solution, while the $S(t)$ contains the time dependency. The number of terms $m$ in the expansion (2) is called number of modes. An alternative direction scheme is adapted to enrich the PGD basis [5].

\section{B. Exploiting superposition principle}

For linear problems with several excitation sources, like multiple coils, the general approach stated above may not converge or produce adverse results. Due to the linear nature of the field problem in absence of nonlinear materials, the superposition of the fields produced by each of the $k$ sources add up to the total field distribution and therefore, an adapted approach is employed (3) [12].

$$
\boldsymbol{A}(\mathrm{x}, \mathrm{t}) \approx \sum_{\mathrm{j}=1}^{k} \sum_{\mathrm{i}=1}^{\mathrm{m}} \boldsymbol{R}_{\mathrm{j}, \mathrm{i}}(x) S_{\mathrm{j}, \mathrm{i}}(t)
$$

Inserting 3 into 1 , leads to a differential algebraic equation for computing the space mode (4) and an ordinary differential equation (5) for the time mode.

$$
\begin{aligned}
& \sum_{\mathrm{j}=1}^{\mathrm{k}} \sum_{\mathrm{i}=1}^{\mathrm{m}} \int_{\mathrm{t}} S_{\mathrm{j}, \mathrm{i}}(t) S_{\mathrm{j}, \mathrm{m}}(t) d t \int_{\mathrm{G}} \nu \nabla \times R_{\mathrm{j}, \mathrm{i}} \nabla \times R_{\mathrm{j}, \mathrm{m}}^{*} d G \\
& +\sum_{\mathrm{j}=1}^{\mathrm{k}} \sum_{\mathrm{i}=1}^{\mathrm{m}} \int_{\mathrm{t}} \frac{\partial S_{\mathrm{j}, \mathrm{i}}(t)}{\partial t} S_{\mathrm{j}, \mathrm{i}}(t) d t \int_{\mathrm{G}} \sigma R_{\mathrm{j}, \mathrm{i}} R_{\mathrm{j}, \mathrm{m}}^{*} d G \\
& =\sum_{\mathrm{j}=1}^{\mathrm{k}} \int_{\mathrm{t}} I_{\mathrm{j}}(t) S_{\mathrm{j}, \mathrm{m}}(t) d t \int_{\mathrm{G}} N_{\mathrm{j}}(x) R_{\mathrm{j}, \mathrm{m}}^{*} d G
\end{aligned}
$$




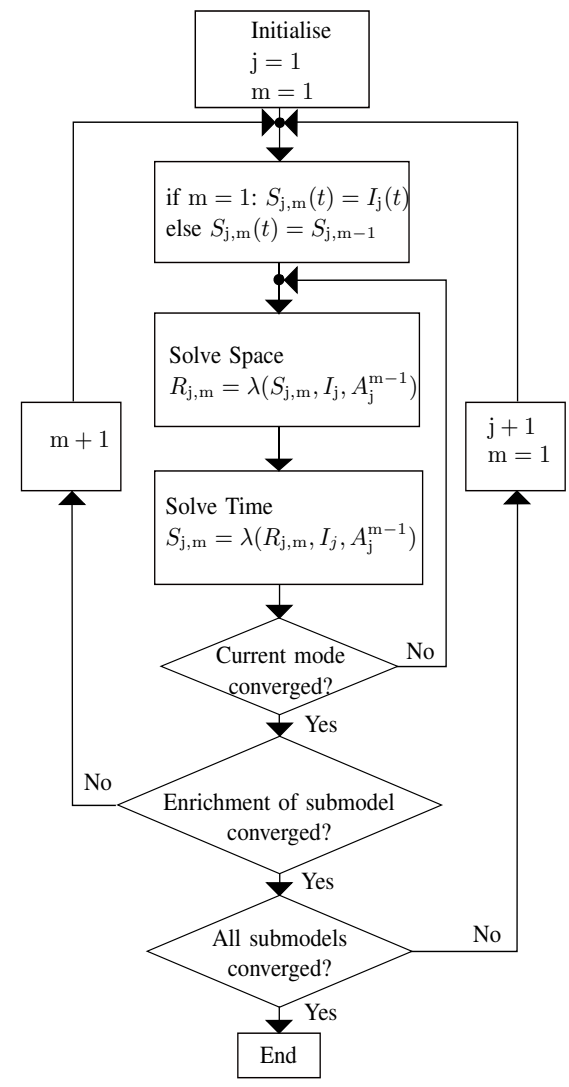

Fig. 1. Alternating scheme to determine linear PGD representation.

$$
\begin{aligned}
& \sum_{\mathrm{j}=1}^{\mathrm{k}} \sum_{\mathrm{i}=1}^{\mathrm{m}} S_{\mathrm{j}, \mathrm{i}}(t) \int_{\mathrm{G}} \nu \nabla \times R_{\mathrm{j}, \mathrm{i}} \nabla \times R_{\mathrm{j}, \mathrm{m}} d G \\
& +\sum_{\mathrm{j}=1}^{\mathrm{k}} \sum_{\mathrm{i}=1}^{\mathrm{m}} \frac{\partial S_{\mathrm{j}, \mathrm{i}}(t)}{\partial t} \int_{\mathrm{G}} \sigma R_{\mathrm{j}, \mathrm{i}} R_{\mathrm{j}, \mathrm{m}} d G \\
& =\sum_{\mathrm{j}=1}^{\mathrm{k}} \int_{\mathrm{t}} I_{\mathrm{j}}(t) S_{\mathrm{j}, \mathrm{m}}(t) d t \int_{\mathrm{G}} N_{\mathrm{j}}(x) R_{\mathrm{j}, \mathrm{m}} d G
\end{aligned}
$$

This leads to the solution of $k$ meta models which need to be analyzed in terms of convergence of the metamodel mode enrichment process as well as the absolute convergence of the full decomposition. The principle is depicted in Fig. 1, which is followed until every submodel $j=[1, k]$ has converged. Due to superposition, the submodels do not depend on each other and can therefore be computed with a fixed $j$ and superposed in the post-processing stage. For stabilizing the enrichment procedure one of the two entities $\boldsymbol{R}$ or $S$ should be normed to prevent divergence, if one entity tends to converge towards zero while the other diverges to infinity. In our case we normed the space modes.

\section{ACCURACY OF THE PGD}

Even though the PGD is applied to many areas, the error evaluation and the information content of the single modes were not a main focus of research. The enrichment is terminated after a certain a-posteriori relative error is fulfilled or until a defined number of modes are enriched [5], [7][9]. To overcome this disadvantage, different error criteria are introduced and compared in this paper.

\section{A. A-Posteriori Error Criteria}

The need for a reference solution, which has to be obtained from the complete system of equations, characterize aposteriori error criteria. Common criteria in this context use the magnetic energy, the Joule losses (6) or the reference solution $\mathbf{X}_{\text {ref }}$ (7) evaluated using the two norm.

$$
\begin{aligned}
\epsilon_{j} & =\frac{\left\|P_{j, r e f}-P_{\mathrm{j}, \mathrm{PGD}}\right\|_{2}}{\left\|P_{\mathrm{j}, \mathrm{ref}}\right\|_{2}} \\
\epsilon_{\text {ref }} & =\frac{\left\|X_{P G D}-X_{\text {ref }}\right\|_{2}}{\left\|X_{\text {ref } f}\right\|_{2}}
\end{aligned}
$$

\section{B. A-priori Error Criteria}

One option to evaluate the relative convergence of the enrichment process is comparing the norm of the difference between the PGD model with $m$ and $m-1$ modes (8)[5][10]. The mentioned criterium is a measure for the relative convergence of the decomposition process, but is missing to give information about the absolute error.

$$
\epsilon_{\mathrm{sol}}=\frac{\left\|X_{P G D}^{m}-X_{P G D}^{m-1}\right\|_{2}}{\left\|X_{P G D}^{m-1}\right\|_{2}}
$$

To retain the a-priori property of the PGD and to cope with disadvantages of [8], two criteria are presented in the following paragraphs. Combining these two leads to a reasonable measure of relative and absolute convergence of the decomposition.

\section{1) Absolute Residual}

Instead of comparing the reference solution to the PGD solution it is more convenient to compute the absolute residual (6). Although, a reference solution is not required, the evaluation of all time steps in (9), with the reference system matrix $\mathbf{M}$ and the time dependent excitation $\boldsymbol{B}_{\mathrm{PGD}}(t)$ is still necessary, resulting in high computational efforts. This criterium can be interpreted as an a-priori version of (7) and yields the absolute residual.

$$
\begin{aligned}
\epsilon_{A b s} & =\frac{\left\|\mathbf{M} \boldsymbol{X}_{\mathrm{PGD}}(t)+\boldsymbol{B}_{\mathrm{PGD}}(t)\right\|_{2}}{\left\|\boldsymbol{B}_{\mathrm{PGD}}(t)\right\|_{2}} \\
\boldsymbol{B}_{\mathrm{PGD}} & =\boldsymbol{J}(t)-\sigma \frac{\boldsymbol{A}_{\mathrm{PGD}}(t-1)}{d t}
\end{aligned}
$$

\section{2) Information content}

An additional approach can be formulated by using the singular value decomposition. Under the assumption that the singular values of the system decrease rapidly, they can be used as a measure of convergence of the enrichment. The 


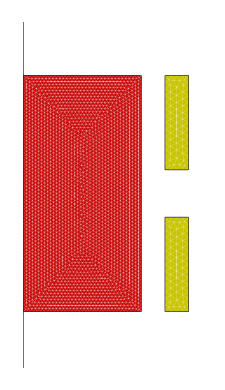

Fig. 2. Transient academic example (Multi Coil Model); magnetizing coils (green), conductive sample (red).

evaluation of the PGD solution in a certain time step can be reformulated into matrix form by

$$
\begin{aligned}
\boldsymbol{A}(x, t) & \approx \sum_{\mathrm{j}}^{\mathrm{k}} \sum_{i=1}^{m} \boldsymbol{R}_{\mathrm{j}, \mathrm{i}}(x) S_{\mathrm{j}, \mathrm{i}}(t) \\
& =\sum_{\mathrm{j}}^{\mathrm{k}} \mathbf{M}_{\mathrm{R}, \mathrm{j}} \cdot \boldsymbol{S}_{\mathrm{j}} .
\end{aligned}
$$

In Eq. (11), $\mathbf{M}_{\mathrm{R}}$ is a matrix with the space modes $\boldsymbol{R}_{\boldsymbol{i}}$ as columns and $\boldsymbol{S}$ is a vector with the values of $S_{i}(t)$ in the evaluation timestep as entries. The matrix $\mathbf{M}_{\mathrm{R}}$ can be decomposed by a singular value decomposition and the resulting singular values give a hint of the information content of the modes, since $\mathbf{M}_{\mathrm{R}}$ acts as a linear projection on $\boldsymbol{S}$. In order to cope with the high computational effort of the SVD, one property of the singular values can be taken advantage of. In order to avoid the high effort of singular value decomposition, the eigendecomposition of the matrix $\mathbf{M}_{R}^{\mathrm{T}} \mathbf{M}_{R}$ can be used.

\section{Simulation}

The previously discussed criteria are applied to a cartesian academic example holding linear material properties. The model consists of a conductive sample in combination with two excitation coils (s. Fig. 2). The conductivity of the sample is equal to $10 \mathrm{kS} / \mathrm{m}$, while the reluctivity of the sample is arbitrary set to $2183.2 \mathrm{Am} / \mathrm{Vs}$. The absolute error tolerance was set to 0.3 per mil. The domain is bound by a dirichlet boundary condition in reasonable distance to the area of interest and at the y-axis. The basic approach (2) is not able to model the transient field distribution for multiple coils, especially if they have a phase lag. Therefore, this model is operated with one sinusoidal fed coil and one cosinusoidal fed coil. The cosinussoidal signal is ramped up in the first fourth of a period. Using the adapted PGD approach (3), leads to a reasonable decomposition. The tolerance regarding the absolute residual for the multicoil model is set to 0.3 per mil. From Fig. 3 it can be depicted that the eddy current losses of the reduced order model (ROM) are in good agreement with the reference solution. The error of the Joule losses is depicted in Fig. 4. The multicoil model needs at least 33 modes to achieve an error 6 smaller than 1\%. Fig. 5 shows the singular values of $\mathbf{M}_{\mathrm{R}}$ (11) and it can be recognized that the singular values decrease rapidly after a certain number

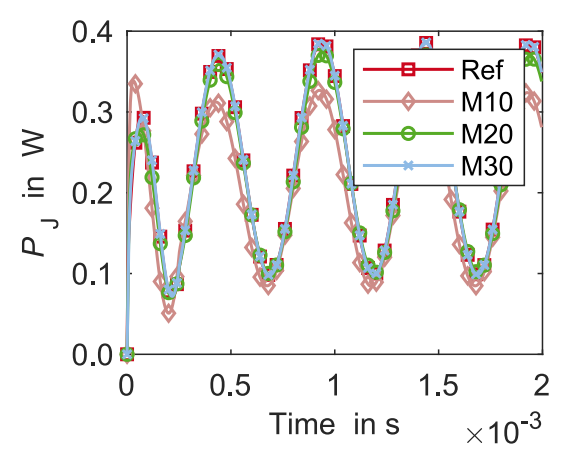

Fig. 3. Eddy current losses in the conductive sample.

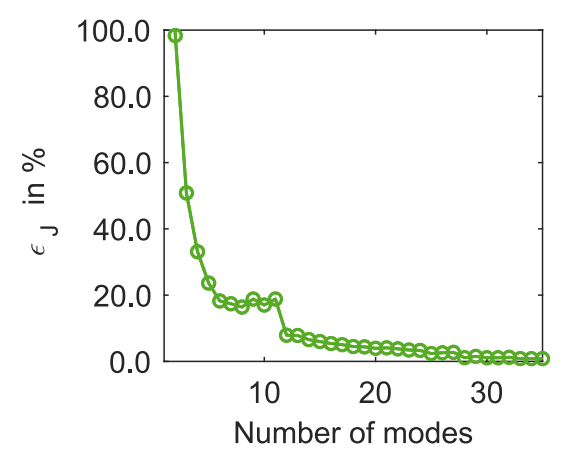

Fig. 4. Relative error of the eddy current losses.

of modes is enriched. The absolute error is depicted in Fig. 6. Comparing the absolute error in Fig. 6 with Fig. 5 shows that the singular values indicate convergence of the enrichment process, but is too coarse to be sufficient on its own, especially for complex models. The absolute mathematical error (9) holds a reasonable error indicator, due to the fact that it analyzes the accuracy of the reduced solution in context of the reference system. To evaluate the mathematical error, the residual has to be built in each time instance and leads to additional computational effort. To diminish this effort, the mathematical error is first evaluated after the singular values significantly decreased $\left(\sigma_{\max } / \sigma_{\min }=0.01\right)$ in comparison to the first. In Fig. 6 it is obvious that the decomposed model is not optimal and converges very slowly. To improve the convergence behaviour, the decomposition can be further improved by using an update step of all time functions, depicted by Multi-U in Fig. 6 [1]. Due to the fact that the MOR extracts most of the relevant information, there is still a certain loss in accuracy compared to the reference in terms of the absolute residual. Further, the PGD does not necessarily have to be orthogonal, in contrast to the POD [1]. Even though the not updated multicoil model produces accurate Joule losses, the absolute residual is quite high. This clearly indicates the difference between comparing Joule losses (6) and the mathematical residual (9). While (6) is a global integrated quantity (9) is the true residual of the system. In addition, it is obvious that the decomposition is crucially improved by the update. Finally the errors computed by (7) and (9) are compared here, because (9) can be interpreted as an a-priory version of (7). 


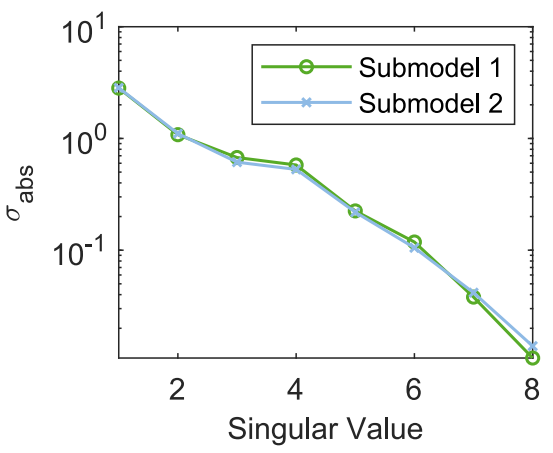

Fig. 5. Singular Values of $\mathbf{M}_{\mathrm{R}}$.

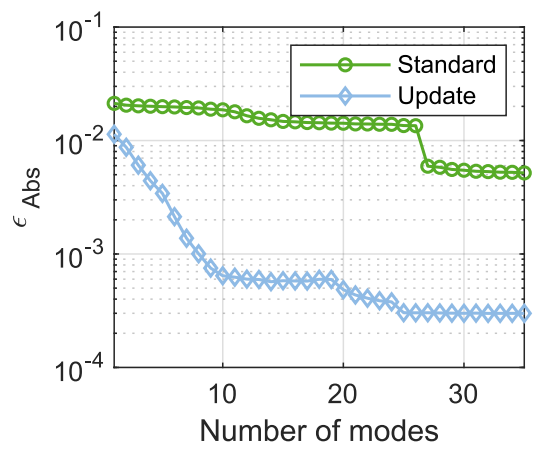

Fig. 6. Absolute Residual Error.

Both errors are depicted in Fig. 7 and it can be seen that the overall behaviour is similar but a certain offset is visible. This deviation originates from the load vector (10), which depends on the decomposition. This leads to the conclusion that (9) can be used as an convergence indicator. The combination of both a-priori criteria are competitive to the a-posteriori criteria in terms of accuracy, but do not need reference solutions and hold less computational effort. The time for the model creation and different error criteria is given in Tab. I, related to the multicoil model. The reference needs $232 \mathrm{~s}$ for the multicoil model, while the PGD enrichment needs $82 \mathrm{~s}$, excluding the calculation of (9).

TABLE I

TIME OF ERRor CRITERIA EVALUATION

\begin{tabular}{|c|c|c|c|c|}
\hline \multicolumn{2}{|c|}{ PGD } & A-Posteriori & \multicolumn{2}{c|}{ A-Priori } \\
\hline Offline & Online & Joule Losses & SVD & Math. Res. \\
\hline $82 \mathrm{~s}$ & $2 \mathrm{~s}$ & $21 \mathrm{~s}$ & $0.1 \mathrm{~s}$ & $52 \mathrm{~s}$ \\
\hline
\end{tabular}

\section{CONCLUSIONS}

A measure for convergence of the enrichment process of the PGD is proposed, which is not based on reference solutions. In combination with (9), the a-priori property of the PGD can be kept, while receiving important information about the relative and absolute convergence. Consecutively, the comparison between commonly used error and convergence indicators points the advantages of the proposed a-priori criteria out.

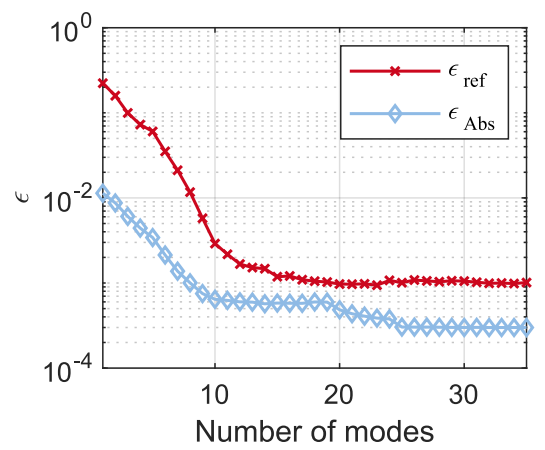

Fig. 7. Comparison of a-priori $\left(\epsilon_{\mathrm{Abs}}\right)$ and a-posteriori $\left(\epsilon_{\mathrm{ref}}\right)$ residual error.

\section{ACKNOWLEDGMENT}

This work was supported by the German Research Foundation (DFG) within the research project number 347941356 "Numerical Analysis of Electromagnetic Fields by Proper Generalized Decomposition in Electrical Machines".

\section{REFERENCES}

[1] A. Nouy, "A priori model reduction through Proper Generalized Decomposition for solving time-dependent partial differential equations" in Computer Methods in Applied Mechanics and Engineering, vol. 199, no. 23, pp. 1603-1626, April 2010.

[2] F. Chinesta, R. Keunings, A. Leygue, The Proper Generalized Decomposition for Advanced Numerical Simulations. A Primer., 1. ed., Octobre 2013, New York: Springer.

[3] M. Nasri, C. Robert, A. Ammar, S. El Arem, F. Morel, "Proper Generalized Decomposition (PGD) for the numerical simulation of polycrystalline aggregates under cyclic loading", Comptes Rendus Mécanique, vol. 346, no. 2, pp. 132-151, 2018.

[4] L. Tamellini, O. Le Maître, A. Nouy, "Model Reduction Based on Proper Generalized Decomposition for the Stoachastic Steady Incompressible Navier-Stokes Equations", SIAM J. Sci. Comput., vol 36, no. 3, pp.1089$1117,2014$.

[5] T. Henneron, S. Clénet, "Model order reduction of quasi-static problems based on POD and PGD approaches", Eur. Phys. J. Appl. Phys., vol. 64, Nov. 2013.

[6] S. Clénet, T. Henneron, "Error Estimation for Model-Order Reduction of Finite-Element Parametric Problems", IEEE Trans. Magn, vol. 52, pp. 1-10, 2016.

[7] Z. Qin, H. Talleb, Z. Ren, "A Proper Generalized Decomposition-Based Solver for Nonlinear Magnetothermal Problems", IEEE Trans. Magn., vol. 52, pp. 1-9, 2016.

[8] Z. Qin, H. Talleb, S. Yan, X. Xu, Z. Ren, "Application of PGD on Parametric Modeling of a Piezoelectric Energy Harvester", IEEE Trans. Magn., vol. 52, pp. 1-11, 2016.

[9] C. Krüttgen, K. Hameyer, "An Efficient Algorithm For Accurate Reduced Models With The Proper Generalized Decomposition For MagnetoquasiStatic Problems", 21st International Conference on the Computation of Electromagnetic Fields, Compumag 2017, https://www.compumag.org/ CMAG2017/[PA-M1-3]_56.pdf.

[10] P. Alotto, M. Guarnieri, F. Moro, A. Stella, "A Proper Generalized Decomposition Approach for Fuel Cell Polymeric Membrane Modeling", IEEE Trans. Magn., vol. 47, no. 5, pp. 1462-1465, 2011.

[11] F. Chinesta, A. Ammar, E. Cueto, "Recent advances and new challenges in the use of the proper generalized decomposition for solving multidimensional models", Arch. Comput. Methods Eng., vol. 17, no. 4, pp. 327-350, 2010

[12] L. Montier, T. Henneron, S. Clénet and B. Goursaud, "Proper Generalized Decomposition Applied on a Rotating Electrical Machine," IEEE Trans. Magn., vol. 54, no. 3, pp. 1-4, March 2018.

[13] T. Henneron, A. Benabou and S. Clenet, "Nonlinear Proper Generalized Decomposition Method Applied to the Magnetic Simulation of a SMC Microstructure," IEEE Trans. Magn., vol. 48, no. 11, pp. 3242-3245, Nov. 2012 . 